Utilisation pragmatique de cartes mentales comme outil d'évaluation en éducation relative à l'environnement

Emmanuel Legrand

\author{
(2) OpenEdition \\ Journals \\ Édition électronique \\ URL : http://journals.openedition.org/ere/6997 \\ DOI : $10.4000 /$ ere.6997 \\ ISSN : 2561-2271 \\ Éditeur \\ Centr'ERE
}

Référence électronique

Emmanuel Legrand, «Utilisation pragmatique de cartes mentales comme outil d'évaluation en éducation relative à l'environnement », Éducation relative à l'environnement [En ligne], Volume 2 | 2000, mis en ligne le 15 septembre 2000, consulté le 17 avril 2021. URL : http://journals.openedition.org/ere/ 6997 ; DOI : https://doi.org/10.4000/ere.6997 


\title{
Utilisation pragmatique de cartes mentales comme outil d'évaluation en éducation relative à l'environnement
}

\author{
Emmanuel Legrand
}

1 Cet article présente l'utilisation de cartes mentales et leur analyse comme technique d'évaluation en éducation relative à l'environnement (ErE) dans le cadre d'une recherche-action menée de 1997 à 2000 en partenariat entre le GREFE (Groupe de Recherche en Éducation et Formation à l'Environnement) et la Haute École de Bruxelles, section pédagogie (Institut d'enseignement supérieur pédagogique De Fré).

2 Une équipe de volontaires, des formateurs (professeurs de sciences, sciences humaines, pédagogie, morale, français, etc.) et quelques futurs instituteurs et régents ${ }^{1}$ en formation initiale (dénommés par la suite "étudiants ») a été constituée à l'Institut De Fré.

3 Dans la première période de travail (janvier 1997 à décembre 1999), la recherche-action poursuivait un double objectif : pour l'Institut De Fré, faire expérimenter des activités d'ErE par les étudiants lors de leurs stages en école primaire ou secondaire; pour le GREFE, construire et tester des méthodes d'évaluation d'activités en ErE, (soutenu par le travail du réseau REVERE). L'objet partagé (Goffin, 1999) de ces deux objectifs était : d'une part, la construction et le test des méthodes d'évaluation s'appuient sur des activités en ErE ; d'autre part, celles-ci exigent une évaluation.

4 Très vite, les partenaires se sont rendu compte qu'il était nécessaire d'évaluer les différents niveaux du partenariat: il ne s'agissait pas seulement d'évaluer les apprentissages des élèves de primaire et de secondaire; il était aussi nécessaire de mesurer l'impact du partenariat GREFE - Institut De Fré sur les conceptions des étudiants. C'est ainsi qu'au début du partenariat, la technique des cartes mentales a été utilisée pour identifier la conception de l'ErE des étudiants. Chaque carte mentale sera comparée à une seconde, effectuée en fin de recherche-action. 
Durant cette première période, les cartes mentales ont été interprétées de manière assez intuitive en référence à la définition de l'environnement comme éco-sociosystème (Goffin, 1998) et à l'aide d'un entretien avec chaque auteur. Cela a fait apparaître à la fois la richesse de la technique et la nécessité de construire des méthodes aidant à analyser les cartes. Si bien que nous avons décidé de réutiliser cette technique pour la deuxième partie de la recherche-action qui débutait en janvier 1999.

Cette deuxième partie consiste en la création d'une véritable formation en ErE pour les régents. La première année de cette formation a commencé en septembre 1999 (un prétest par cartes mentales a été effectué) et se terminera en juin 2000. Le groupe se composant d'une quarantaine de personnes, il était impossible de reprendre la méthode intuitive utilisée auparavant. Le GREFE a donc décidé d'actualiser le corpus de cartes mentales de la première partie de la recherche-action afin de proposer des méthodes d'analyse efficaces pour un grand groupe et d'approfondir nos intuitions de départ. L'objectif est de construire ces méthodes avant le post-test de la fin de l'année scolaire 2000.

7 Après avoir expliqué brièvement la technique des cartes mentales et son fondement scientifique, nous montrerons en quoi elle peut être utilisée pour l'ErE et en particulier pour l'évaluation en ErE. Enfin, nous proposerons nos méthodes d'analyses.

\section{La technique des cartes mentales}

La technique des cartes mentales se présente généralement selon le principe suivant: un concept dont on veut percevoir le sens par un individu est proposé au centre d'une feuille vierge. Partant de ce mot-stimulus, la personne évaluée est invitée à évoquer et placer en étoile les mots qu'elle lui associe, en les reliant à l'aide de flèches. On crée ainsi un réseau de concepts ${ }^{2}$.

Cette technique permet « de mettre en évidence non seulement les connaissances que possèdent les apprenants sur un sujet donné, mais aussi comment celles-ci sont interreliées dans la mémoire à long terme » (Iguanane, Marchand et d'Ivernois, 1999). Elle permet d'embrasser la signification d'un concept pour un individu de façon plus large et plus concrète que par l'énoncé d'une définition formelle. Un grand classique en ErE est de créer une carte mentale autour du mot « environnement » en accompagnant le mot-stimulus d'une consigne verbale du type: "pour vous, que recouvre le terme environnement?». La figure 1 qui suit présente un exemple de ce type de carte mentale.

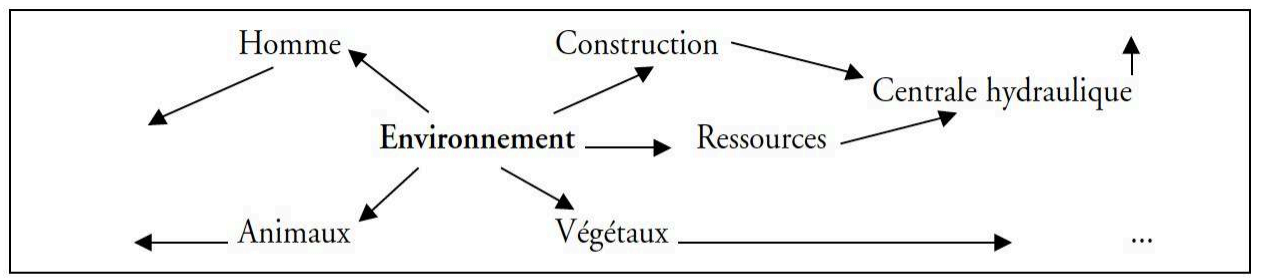

Figure 1 : Exemple de carte mentale de l'environnement

10 Par expérience, cette technique est adaptée dès l'enseignement secondaire, bien que notre équipe l'ait déjà utilisée en primaire. Lorsqu'on l'utilise, il est souvent utile de 
donner un exemple de carte mentale portant sur un autre sujet afin de familiariser la personne avec le principe sans risquer d'induire ses concepts.

\section{Le fondement scientifique}

11 Le principe des cartes mentales pour l'évaluation des connaissances est basé sur les théories de l'apprentissage, en particulier sur le courant cognitiviste (Gardner, 1993). Ce courant ne conçoit pas la pensée humaine comme une suite linéaire d'idées classées en une série d'embranchements successifs, mais comme un système, un réseau conceptuel. Johnson-Laird (1983) distingue les modèles conceptuels conçus par le formateur et les modèles mentaux réellement utilisés par les apprenants. La carte mentale serait un moyen de visualiser le modèle mental considéré comme un système complexe de relations entre les concepts. Cette théorie du réseau conceptuel contrebalance la conception béhavioriste de l'apprentissage qui considère l'apprenant comme vierge de toute idée (Albertini, 1985). Tout apprenant a un modèle mental, même très restreint, dont il faut tenir compte lors de l'apprentissage. Il est dès lors indispensable d'identifier les connaissances préalables de l'apprenant afin d'y " accrocher les nouvelles notions aptes à enrichir le réseau conceptuel préexistant " (Norman, 1982).

12 Selon Marchand (1997), les cartes mentales :

- permettent d'extérioriser la pensée des individus, c'est-à-dire les concepts à partir desquels de nouvelles connaissances vont être acquises,

- mettent en évidence les connaissances erronées et/ou partielles etc.,

- visualisent comment les apprentissages se réalisent par la représentation des relations de sens entre les concepts.

13 À ces trois éléments, il faut ajouter que les réseaux conceptuels sont de nature plus complexe qu'une série ordonnée de savoirs, rangés sous forme de définitions précises dans notre mémoire. Le tracé de cartes mentales permet d'embrasser non seulement la complexité de notre pensée et, mais aussi la complexité de l'environnement. À notre sens, cette technique a, en ErE, la même légitimité épistémologique que les méthodes de schématisation systémique telles que Ragou (2000, p.60) les approfondit dans sa thèse doctorale sur la didactique de l'approche systémique à destination des enseignants. Pour légitimer son étude, l'auteur se base sur l'existence "d'un large consensus pour rejeter ce qui serait un cours d'environnement et prôner le plus possible des études de cas concrets dont la complexité apparaît vite ${ }^{3} »$.

\section{Les cartes mentales en ErE}

\section{Des cartes mentales, pour quels objectifs d'ErE?}

En pédagogie, les cartes mentales sont principalement utilisées pour évaluer les connaissances et/ou la compréhension-intégration des connaissances. Or, augmenter la connaissance et la compréhension des connaissances est l'objectif numéro deux de l'ErE selon l'UNESCO (1978).

En outre, dans le cas précis de l'ErE, les cartes mentales peuvent aussi apporter leur aide pour évaluer la prise de conscience des problèmes environnementaux. En effet, les 
cartes mentales permettent de repérer les connaissances manquantes. Si une carte mentale construite autour du concept «environnement» ne comportait pas de références aux problèmes environnementaux, mais seulement des termes comme: nature, promenade, etc., on pourrait s'inquiéter de la sensibilité actuelle de son auteur à cet environnement !

Mais, selon l'UNESCO (1978), la prise de conscience et l'acquisition de connaissances ne sont que les deux premiers objectifs de l'ErE. Dès Tbilissi (1977), il a fallu les envisager comme un passage obligé pour arriver à un changement d'état d'esprit et, mieux encore, à un changement de comportement. Cependant, il est souvent difficile de mettre en place un système d'observation du changement effectif des comportements.

Par exemple, des changements de comportements en matière de gestion des déchets scolaires sont certes évaluables directement en prenant la mesure des déchets euxmêmes (diminution des quantités rejetées, augmentation des quantités triées, augmentation de la qualité du tri). En revanche, on peut difficilement imaginer de suivre chaque élève pour vérifier s'il a acquis les comportements adéquats, de manière permanente, à l'école et en dehors de celle-ci. Même si l'occurrence des comportements cibles était mesurée, rien ne prouve qu'ils seraient produits avec conviction et non par soumission à l'autorité ou pour d'autres raisons.

18 Cette idée de "conviction » est reprise dans le concept "d'attitude » défini comme « une disposition déterminée par l'expérience qui exerce une influence directrice sur la conduite " (Lafon, 1979). Différents tests, plus ou moins sophistiqués, essayent d'évaluer ces «attitudes» qui reposent sur au moins deux éléments distincts quoiqu'inséparables :

- Le premier est de l'ordre de la motivation : " être sensible à », " vouloir agir de telle manière à » ou mieux " vouloir convaincre les autres d'agir», aspect qui est souvent envisagé au travers de typologies de degrés, d'intensité d'attitudes telles que celles de Krathwohl et coll. (1964), Gagne (1965), etc.

- Le second est d'ordre conceptuel : quels sont les contenus, les objets ? où s'appliquent ces degrés d'intensité (par exemple, le respect des plantes, des êtres humains, de la nature ; la gestion des ressources) ? Ces aspects conceptuels du changement d'attitude - autre objectif d'ErE - peuvent aussi être évalués par les cartes mentales.

\section{Une possibilité d'utilisation des cartes mentales pour l'évaluation en ErE}

19 L'utilisation de la carte mentale peut se justifier pour l'évaluation en ErE non seulement comme prétest et post-test d'une formation, mais aussi pour préciser les objectifs de cette formation. En effet, il s'agit tout d'abord de faire construire des cartes mentales par le public cible avant une formation (le prétest). Ensuite, la construction de la formation se basera sur les éléments "non - ou mal - envisagés» dans les cartes mentales. Enfin, une séance de construction de cartes mentales (le post-test) permettra de déceler l'enrichissement, l'accrochage conceptuel ainsi obtenu.

Lors de la deuxième partie de la recherche-action avec l'Institut De Fré, les cartes mentales du prétest ont fait apparaitre autour de l'acronyme « ErE » des concepts qui représentent surtout la nature: animaux, forêt, arbre, ainsi que des mots comme pollution, déchets, chasse (les nuisances). La formation en ErE consécutive au prétest s'est orientée vers diverses activités aptes à élargir la conception de l'environnement 
afin qu'elle ne soit pas basée sur la seule notion de nature et de la protection de celle-ci, mais, également, sur les aspects anthropiques. Pour démontrer un accrochage de nouvelles notions, le post-test qui aura lieu en juin 2000 devrait comporter des mots tels que maison, rue, aménagement du territoire, réserve naturelle, aire de stationnement, aire de repos, etc.

\section{Proposition de méthodes d'analyses}

L'analyse des éléments d'une carte mentale peut, cependant, poser certains problèmes :

- Une carte mentale peut contenir entre 10 à 70 mots multipliés par 5 à 25 participants. Répertorier les mots, calculer leur fréquence, prend du temps. Dans le cadre d'un court module de formation (par exemple, une semaine), ce serait difficilement réalisable, d'autant que ce travail doit également être effectué pour le post-test. La grande quantité d'informations à traiter peut donc constituer un obstacle à l'utilisation de cette technique pour des formations courtes.

- Il existe certes des méthodes d'analyses de cartes mentales qui via un traitement informatique permettent l'analyse des données, qui les affinent même, par exemple, en calculant la distance entre les mots. Cependant, leur lourdeur technique et la complexité des modèles d'analyse les rendent quasi inaccessibles à un praticien en ErE.

- L'analyse d'une carte mentale ne peut se contenter d'un simple comptage de mots qui ne tiendrait pas compte de leurs relations. Il suffirait alors de demander au public une simple liste et pas une carte mentale! Il s'agit d'employer des procédures qui conservent les informations de type systémique, par exemple, en comptant le nombre de relations entre les éléments de la carte.

Dans le cadre de l'évaluation d'une courte formation en ErE, il s'agit donc de trouver des méthodes d'analyse qui, tout en allégeant les modes de calcul, soient aptes à évaluer cet enrichissement de mots et de liens. Le nœud du problème se trouve bien dans la façon dont est traduit, en indicateurs, le concept d'enrichissement entre le prétest et le post-test.

3 Imaginons un instituteur qui prendrait comme sujet : sensibiliser les élèves à la nature. Ceux-ci pour la décrire donneront certainement divers noms de vertébrés et divers noms d'arbres et de fleurs. Cet instituteur peut estimer qu'il a enrichi leur conception de la " nature » si, en fin de cours, les élèves peuvent citer une plus grande quantité de noms d'arbres, de fleurs. Il pourrait penser avoir encore mieux réussi si les élèves regroupent ces éléments en catégories et ajoutent celles des champignons, des insectes, des invertébrés. Ce saut qualitatif (nouvelles catégories et organisation de celles-ci) marque un enrichissement de concept plus intéressant.

Autrement dit, dans ce cadre, utiliser comme indicateur d'enrichissement conceptuel l'augmentation du nombre de catégories sémantiques ${ }^{4}$ recouvertes par les divers mots proposés constitue une meilleure définition, une meilleure «opérationnalisation » de l'enrichissement que de simplement considérer l'augmentation du nombre de mots entre le prétest et le post-test. En outre, comme il est nécessaire d'étudier les relations entre les éléments de la carte, l'aspect systémique, il nous paraît plus pertinent d'évaluer l'augmentation du nombre de relations intercatégories que de compter le nombre total de relations. 
Dans la suite de cet article, nous ferons successivement des propositions pour l'analyse des aspects sémantiques (quelle catégorisation de mots utiliser et comment les analyser?) puis pour l'analyse des aspects systémiques (quelles relations rechercher et comment les analyser?)

\section{L'enrichissement des catégories sémantiques}

\section{Quelles catégories utiliser?}

Les catégories sémantiques relatives à l'ErE peuvent être construites par chacun en fonction du contexte et de ses objectifs. Il est cependant opportun d'utiliser des catégories sémantiques validées par la recherche ou par des modèles théoriques de référence. Le tableau 1 qui suit propose des catégories sémantiques spécifiques à l'ErE qui nous paraissent intéressantes.

Notons que le travail par catégorie sémantique permet, en outre, de ne pas répertorier tous les mots $^{5}$. Il suffit pour chaque individu de comptabiliser le nombre de mots par catégorie étudiée. En outre, il est préférable de laisser une catégorie pour les mots inclassables.

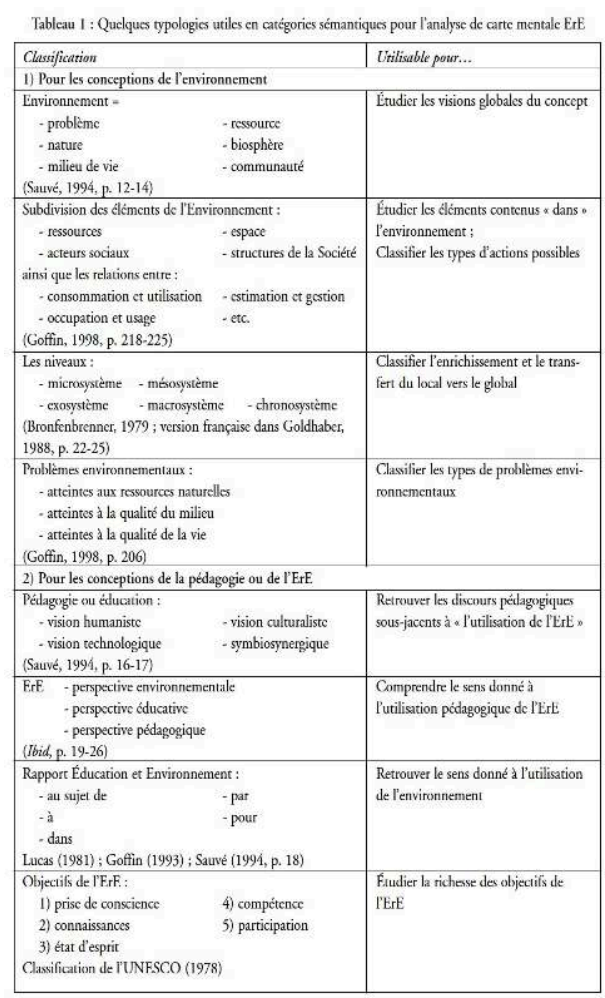

28 Dans la carte mentale ci-dessous (figure 2), nous avons classé les différents concepts en fonction de la subdivision des éléments de l'environnement selon Goffin (1998, p. 222) : Ressources, Espace, Acteurs sociaux, Structures de la société ${ }^{6}$. Une lecture attentive des définitions de ces mots permettra de comprendre le classement proposé. Pour le besoin de la démonstration, nous proposerons la liste des mots pour chaque catégorie. En réalité, nous nous contentons de barrer les mots sur la carte et de les comptabiliser pour la catégorie adéquate.

Il nous paraît important de signaler que l'utilisation de catégories sémantiques définies à l'avance ne supprime pas toute subjectivité. D'abord parce que certains mots peuvent 
ne pas être classés, ensuite parce que d'autres sont ambigus et nécessitent une interprétation. Cette interprétation peut se baser sur l'analyse des catégories auxquelles appartiennent les mots qui lui sont reliés. Dans l'exemple, le mot «famille » peut très bien appartenir à la catégorie "acteurs sociaux" ou à la catégorie "structures de la société ", mais il a été placé dans la première, car il est entouré de mots qui y sont eux-mêmes classés. Autre exemple d'ambiguïté, le mot "communication » peut prendre de nombreuses significations. Comme il se trouvait placé au sein de différents concepts relatifs à la "culture", il a pris place dans la catégorie "structures de la société ». Le mot peut donc prendre sens en fonction du réseau conceptuel où il est inséré. Par ailleurs, nous n'avons pas classé le mot " projet ", car il nous paraît relever de la catégorie sémantique « pédagogie » et nous n'avons pas utilisé cette catégorie.

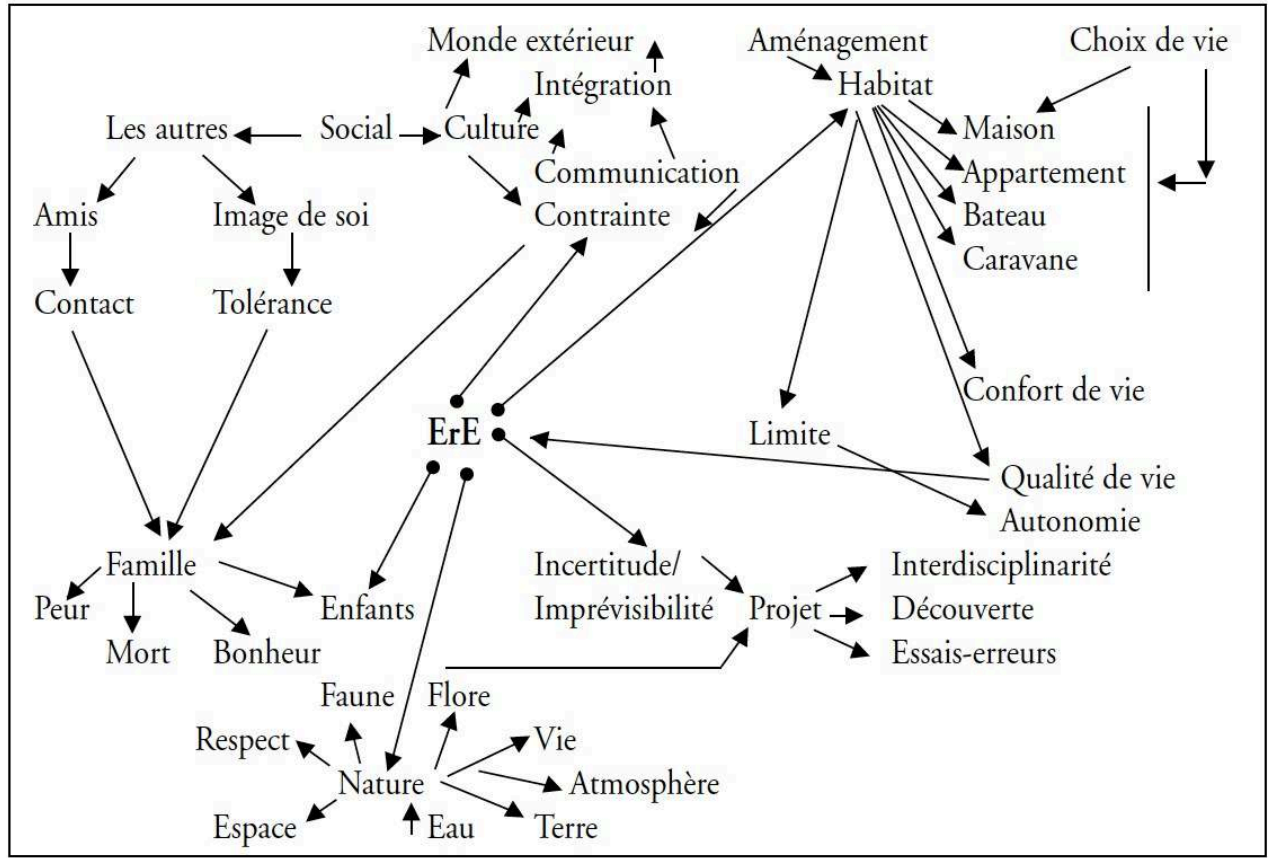

Figure 2 : Exemple de carte mentale de l'ERE

Le classement sera le suivant :

- Les ressources (8 mots) : faune, flore, eau, terre, vie, atmosphère, respect (noter que le terme " énergie » n'est pas développé dans les mots de la carte) ;

-L'espace (11 mots) : espace, nature, habitat, maison, appartement, bateau, caravane, monde extérieur, aménagement, limite ;

- Les acteurs sociaux (12 mots) : famille, enfants, les autres, amis, confort de vie, qualité de vie, autonomie, choix de vie, image de soi, peur, mort, bonheur, contact, tolérance, social (sous-système « implications bio- et psychosociales »);

- Les structures de la société (6 mots) : incertitude, prévisibilité, interdisciplinarité, essaiserreurs, découverte (sous-système de technique) culture, communication, intégration, contrainte (sous-système de culture) ;

- Non classé (1 mot) : projet. 


\section{Comment analyser les cartes ?}

31 Un tableau donnera une vue d'ensemble du groupe. Il reprendra, par exemple, les catégories sémantiques en colonne et les individus en ligne. Dans le tableau $2 \mathrm{du}$ point 4.3, différents indices statistiques seront repris. Voici une description et justification de ces indices.

\section{Présence des catégories}

Comment se faire une idée moyenne des différentes cartes mentales afin d'évaluer l'enrichissement sémantique entre le prétest et le post-test? L'indice le plus intéressant ne serait, ni la moyenne du nombre de mots par carte, ni la moyenne du nombre de mots par catégorie, mais la fréquence d'individus par catégorie. Les questions qui se posent en terme d'enrichissement sont « combien d'individus ont au moins un élément dans telle catégorie? Quelles sont les catégories que le groupe n'envisage pas du tout? » Il est préférable d'utiliser des pourcentages pour exprimer cette fréquence si le nombre d'individus participant au prétest et au post-test n'est pas le même.

Pour évaluer l'enrichissement, nous n'utiliserons pas la moyenne du nombre de mots par réseau, car le réseau n'est pas nécessairement plus riche si cette moyenne s'élève. Il nous faudra vérifier si ce saut est qualitatif. L'analyse de la variété de catégories sémantiques utilisées est donc essentielle pour considérer cet enrichissement. (Il arrive parfois que le nombre de mots diminue lors du post-test, lassitude des sujets, etc.)

La moyenne du nombre de mots par catégorie apporterait une information tronquée : une moyenne très basse indiquerait que cette catégorie est peu utilisée en général, mais une moyenne élevée ou médiante pourrait masquer le fait que certaines personnes n'utilisent pas cette catégorie. Elle ne nous renseignerait que de façon globale sans tenir compte des variations individuelles. Cette moyenne n'est intéressante qu'à condition d'avoir une idée au préalable de la fréquence d'utilisation de la catégorie par les individus.

Comment savoir si le groupe est homogène pour chaque catégorie? L'homogénéité sémantique intracatégorie du groupe pourra être observée à partir de la répartition du nombre d'individus dans chaque catégorie et pas à travers l'écart type du nombre de mots par réseau comme cela se fait parfois. Un groupe serait homogène si la catégorie rassemble un nombre très élevé ou un nombre très petit d'individus. Personnellement, nous travaillons avec les seuils suivants : si plus de $80 \%$ ou moins de $20 \%$ d'individus évoquent telle catégorie, nous le considérons comme homogène.

L'utilisation des écarts types du nombre de mots par réseau ne nous paraît pas traduire l'homogénéité de façon judicieuse. En effet, un groupe peut utiliser un nombre très différent de mots (et donc avoir un grand écart type) alors que tous les mots désignent le même type de réalité, tandis qu'un autre groupe peut utiliser en moyenne le même nombre de mots (et donc un écart type très réduit) qui recouvrent des réalités très différentes. Tout comme pour le calcul des moyennes, il est plus intéressant de calculer les écarts types pour chaque catégorie. Nous le ferons plus loin.

On peut procéder ainsi catégorie par catégorie, mais il serait mieux de comparer les individus entre eux par rapport à leur type de réponses pour toutes les catégories afin de déterminer une homogénéité sémantique intercatégories. Mais ce type d'analyse 
exige des traitements statistiques complexes ${ }^{7}$ qui ne cadrent pas avec une utilisation pragmatique des cartes. L'utilisation des écarts types par catégorie donnera un moyen de contourner la difficulté.

\section{Importance des catégories}

Comme les fréquences ont été calculées uniquement sur la présence d'au moins un mot de la catégorie pour chaque individu, cela empêche d'évaluer l'importance, l'intensité réelle des catégories. La question que l'on se pose est : de quelle façon cette catégorie présente chez autant de personnes - est-elle représentée dans les diverses cartes mentales? Les indices statistiques précédents traitaient uniquement de l'importance d'une catégorie en fonction de sa simple présence (au moins un mot). Ils seront considérés comme des indices absolus tandis que les suivants seront appelés des indices d'intensité :

- La moyenne du nombre de mots employés par les individus dans chaque catégorie permettra de mesurer l'intensité de la richesse sémantique et donc d'affiner notre jugement quant à la richesse relative des catégories.

- Comme les personnes qui se singularisent fortement du reste du groupe (utilisent peu ou pas une catégorie contrairement au reste du groupe) modifient artificiellement la moyenne, on peut décider de nuancer le jugement d'intensité précédent en employant le mode : c'est-àdire le nombre le plus fréquent de mots pour telle catégorie.

- L'écart type sur le nombre de mots à l'intérieur de chaque catégorie affinera le jugement sur le groupe en terme d'homogénéité d'intensité sémantique : un écart type réduit dans une catégorie renseigne sur une plus grande homogénéité. (C'est une mesure plus fine que l'écart type sur le total des mots de chaque réseau.)

\section{L'enrichissement systémique (analyse des relations)}

L'évaluation de la richesse sémantique en terme de présence et/ou d'intensité des catégories pourra être nuancée par un jugement en terme de richesse systémique. Il est possible d'étudier l'organisation des concepts à travers deux méthodes :

La première méthode est quantitative. Il s'agit de comptabiliser dans chaque réseau, non pas le nombre total de liens, mais le nombre de liens intercatégories, c'est-à-dire les liens qui relient des mots d'une catégorie à une autre, à l'exception des liens qui relient un mot avec le mot-stimulus. La logique est la suivante : plus le nombre de liens intercatégories est élevé, plus on pourra estimer que le réseau est riche en relations.

Le nombre total de liens ne donnerait pas d'indication réelle sur l'aspect systémique du réseau. En effet, une personne qui citerait beaucoup d'éléments relevants d'une seule catégorie aurait un nombre élevé de liens pour une organisation trop simple de sa carte mentale.

Mais l'utilisation du nombre de liens intercatégories n'est pas parfaite parce qu'une personne peut établir de nombreux liens intercatégories pour deux catégories et une autre, utiliser cinq ou six catégories sans les relier! Pour tenir compte du nombre de catégories, le nombre de liens intercatégories sera pondéré en utilisant le quotient: nombre de catégories / nombre de liens inter-catégories

Plus le quotient est proche de 0 , plus la richesse systémique sera élevée et inversement, plus le nombre s'élève au-dessus de 1 , plus est établie une pauvreté systémique. 

l'aspect systémique nécessiterait une véritable interprétation des cartes conceptuelles. Comment sont-elles organisées? Il s'agit de repérer les formes de hiérarchisation ou structuration entre les concepts. En effet, selon Johssua et Dupin (1993), «le savoir de l'expert apparaît à la fois comme organisé, mis en structure et fortement hiérarchisé ». Si les éléments sont placés en rayon autour du motstimulus, cela peut indiquer que les différents éléments du concept étudié sont compris indépendamment les uns des autres!

Bien que l'on puisse espérer que l'interprétation de l'organisation d'un réseau soit une analyse plus fine que le comptage du nombre de relations, on peut craindre d'être subjectif en effectuant cette opération. Comment diminuer cette part de subjectivité ? Tout d'abord, il est possible de consulter l'initiateur du réseau pour se faire expliquer certains éléments. Mais cette analyse quasi clinique est longue pour un grand groupe. Ensuite, nous pensons qu'il est possible de répertorier certains indicateurs facilement identifiables de la qualité systémique d'une carte mentale.

Partant de travaux antérieurs sur l'analyse de cartes mentales (Legrand, 1997), nous distinguons trois types de configuration qui témoignent d'une hiérarchisation et structuration et qui sont assez facilement repérables sur les cartes mentales. Nous les avons nommées: nœuds décentrés, embranchements typologiques et relations circulaires. Nous illustrerons ces types par des exemples de cartes mentales issues de la recherche-action à l'Institut De Fré.

- Nœuds décentrés: un concept, autre que le mot-stimulus, est lui-même le centre de différents concepts (figure 3). En psychologie cognitive, les différents éléments qui constituent les cartes mentales ou les classements hiérarchiques linéaires sont appelés nœuds. Nous avons repris ce terme en lui ajoutant le mot « décentré » afin de souligner qu'il prend à lui seul une partie de la signification du concept central par analogie avec le concept de pouvoir décentralisé.

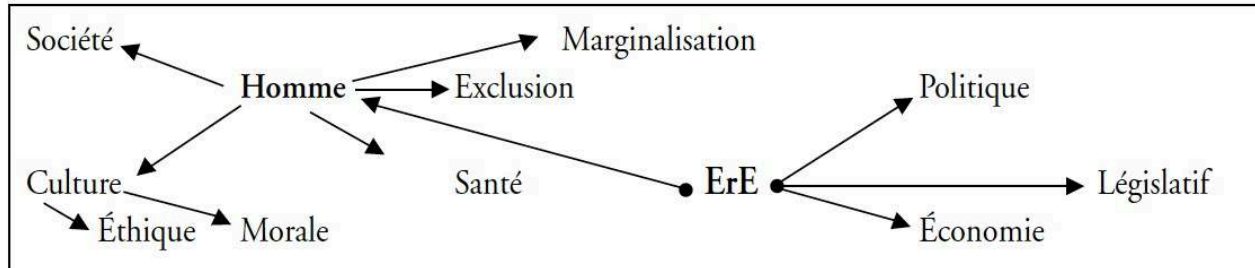

Figure 3 : Exemple de carte mentale à nœuds décentrés

- Embranchements typologiques ${ }^{8}$ : des concepts autres que le mot-stimulus, sont le point de départ de différents éléments ; ils constituent ainsi des sous-catégories (figure 4).

Éducation relative à l'environnement, Volume 2 | 2000 


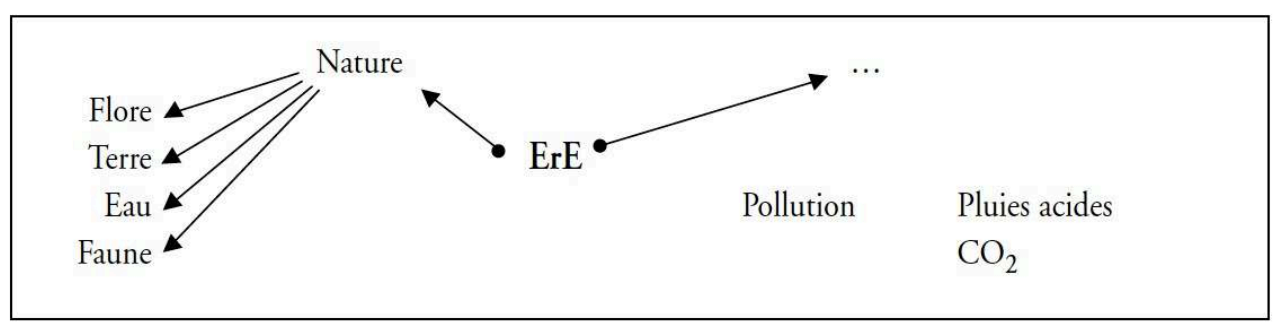

Figure 4 : Exemple de carte mentale à embranchements typologiques

- Relations circulaires : le système des relations est organisé en boucle (un élément est relié à un autre, celui-ci à un troisième, ce dernier à un quatrième, etc. et retour au premier, voir la figure 5). Nous n'avons pas employé le terme de «causalité circulaire " habituel en systémique, car dans les cartes analysées, les relations en boucle ne présentaient pas toujours de causalité. Nous avons donc adopté une appellation moins exigeante.

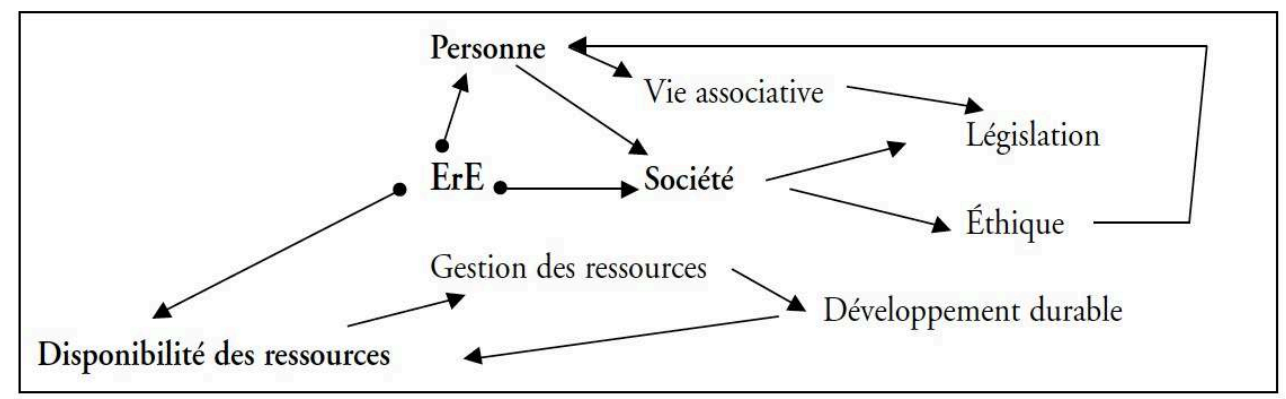

Figure 5 : Exemple de carte mentale à relations circulaires

Pour résumer les analyses interprétatives des différentes cartes mentales, la dernière colonne du tableau permet d'inscrire le nombre total de configurations systémiques repérées en regard de chaque individu.

Pour pouvoir comparer l'ensemble des cartes du prétest à celles post-test, il est intéressant de calculer la moyenne et l'écart type du nombre de configurations; il en sera de même avec les nombres de relations intercatégories et les quotients pondérateurs.

\section{Comparaison prétest et post-test}

Les deux tableaux d'analyse des prétest et post-test (tableau 2) permettent d'étudier les enrichissements sémantiques et systémiques apportés par la formation.

\section{Enrichissement sémantique}

On notera l'apparition de nouvelles catégories dans les cartes mentales et l'augmentation de la fréquence d'individus qui utilisent chaque catégorie. Mais, plus une catégorie est utilisée, au départ, par un nombre important d'individus, moins la probabilité de son augmentation est plausible. On peut recourir au calcul du gain relatif pour chaque catégorie sémantique : 


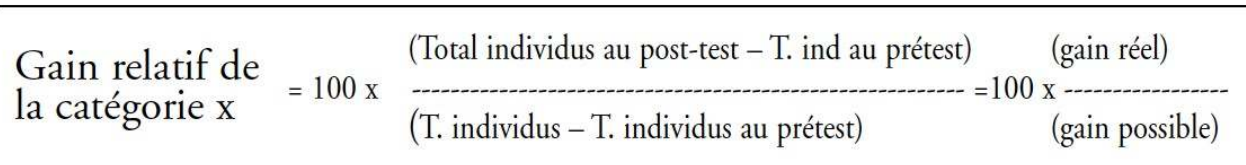

51 Grâce aux indices absolus correspondants, le changement d'ordre dans le classement des catégories peut aussi être observé. Mais si toutes les catégories étaient reprises dès le début, il faudrait utiliser les indices d'intensité (moyenne et mode) pour pouvoir juger de l'évolution des conceptions sémantiques.

Les indices d'homogénéité permettent, quant à eux, de s'assurer que l'enrichissement sémantique n'est pas le fruit de quelques rares bons éléments, que cet enrichissement est bien réparti dans le groupe.

Tableau 2 : Tableau d'analyse des cartes mentales pour le prétest ou le post-test

\begin{tabular}{|c|c|c|c|c|c|c|c|c|}
\hline \multirow{3}{*}{ 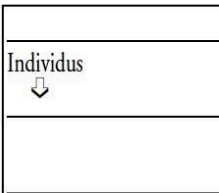 } & \multicolumn{5}{|c|}{ Richesse sémantique } & \multicolumn{3}{|c|}{ Richesse systémique } \\
\hline & \multicolumn{5}{|c|}{ Catégories sémantiques } & \multirow{2}{*}{$\begin{array}{l}\text { Nb. liens inter- } \\
\text { catégories }\end{array}$} & \multirow{2}{*}{$\begin{array}{l}\text { Quotient: } \frac{\mathrm{Nb} . \text { cat. }}{\mathrm{Nb} \text {. liens inter-cat }} \\
\end{array}$} & \multirow{2}{*}{$\begin{array}{l}\begin{array}{l}\mathrm{Nb} . \mathrm{de} \\
\text { configuration }\end{array} \\
\end{array}$} \\
\hline & $\mathrm{CS} 1$ & CS2 & CS3 & CS4 & $\begin{array}{l}\text { non- } \\
\text { classés }\end{array}$ & & & \\
\hline Individu $\mathrm{n}^{0} 1$ & 4 & 5 & 0 & 1 & $\ldots$ & & & \\
\hline Individu $\mathrm{n}^{\circ} 2$ & 3 & 8 & 0 & 2 & $\ldots$ & & & \\
\hline & & & & & & & & \\
\hline$\ldots$ & & & & & & & & \\
\hline & & & & & & & & \\
\hline Individu $\mathrm{n}^{\circ} 25$ & & & & & & & & \\
\hline Total des individus qui & & & & & & Moy. & & \\
\hline ont 1 mot au moins & & & & & & Écart & & \\
\hline dans cette catégorie & & & & & & Type & & \\
\hline Fréquence (en \%) & $50 \%$ & $90 \%$ & $0 \%$ & $10 \%$ & & & \multicolumn{2}{|c|}{ Richesse sémantique absolue } \\
\hline Homogénété & oui & ouix) & (OIII) & (oiix) & & $>80 \%$ ou $<20 \%$ & \multirow{2}{*}{\multicolumn{2}{|c|}{$\begin{array}{l}\text { Homogénéité sémantique } \\
\text { intracatégorie absolue }\end{array}$}} \\
\hline des fréquences & (nom & non & non & non & & $20 \%<f<80 \%$ & & \\
\hline $\begin{array}{l}\text { Moyenne des nombres } \\
\text { de mots/catég. }\end{array}$ & & & & & & & \multicolumn{2}{|c|}{ Intensité de la richesse sémantique } \\
\hline $\begin{array}{l}\text { Mode des nombres } \\
\text { de mots/catég. }\end{array}$ & & & & & & & \multicolumn{2}{|l|}{ Idem } \\
\hline $\begin{array}{l}\text { Écart type sur le nombre } \\
\text { mots/catég. }\end{array}$ & & & & & & & \multicolumn{2}{|c|}{$\begin{array}{l}\text { Homogénéité de l'intensité de la } \\
\text { richesse sémantique }\end{array}$} \\
\hline
\end{tabular}

\section{Enrichissement systémique}

Pour une vision globale, il suffit de comparer entre le prétest et le post-test la moyenne des quotients "nombre de catégories utilisées/nombre de relations intercatégories ». On peut faire de même avec la moyenne des nombres de configurations systémiques. Y a-t-il eu augmentation? Tout en vérifiant l'écart type pour avoir une estimation de l'homogénéité de ces moyennes ${ }^{9}$.

\section{Conclusion}

Premièrement, il est important de bien comprendre qu'il s'agit, ici, d'une mesure d'efficacité du module de formation lui-même, et non pas du travail de chaque participant puisque les indices statistiques proposés comparent l'ensemble des cartes mentales du prétest à l'ensemble des cartes mentales du post-test. Ces indices 
statistiques ne permettent donc pas de se faire une opinion sur chaque individu, ni de le noter.

Deuxièmement, nous voudrions insister sur le fait qu'il s'agit là d'une technique parmi d'autres pour rendre compte de la complexité systémique, et que la carte mentale est un moyen à mettre en œuvre pour l'évaluer. Il est souhaitable de recouper les informations ainsi obtenues avec d'autres observations.

6 Troisièmement, l'analyse sémantique que nous proposons se base sur une conception préalable des catégories à étudier selon la différence entre cartes mentales et cartes conceptuelles telle que proposée par Johnson-Laird (1983). En revanche, les méthodes proposées pour l'analyse systémique ne se fondent pas sur cette adéquation entre un modèle prédéfini par l'évaluateur et ceux proposés par les apprenants. En effet, le fait de comptabiliser les relations intercatégories et/ou les configurations systémiques des cartes ne signifie pas que l'évaluateur soit d'accord avec les relations repérées. Celui-ci ne juge pas la pertinence des relations établies par l'apprenant. Il s'agit là d'une limite importante de ces méthodes. D'un côté, l'analyse sémantique peut être vue comme basée sur un postulat positiviste, tandis que l'analyse systémique serait perçue comme basée sur un postulat relativiste. Plus loin, nous évoquerons des méthodes plus socioconstructivistes.

Quatrièmement, puisque les indices comparent l'ensemble des cartes mentales du prétest à l'ensemble des cartes mentales du post-test (et non chaque individu avec luimême tout au long de notre recherche-action), nous nous sommes rendu compte qu'opérer une différenciation conceptuelle entre « techniques d'évaluation » et « outils d'évaluation » pourrait se révéler très heuristique. L'utilisation des cartes mentales peut être une technique, une méthode d'évaluation assez générale : faire construire des cartes mentales par les participants et les analyser ensuite. Cette technique d'évaluation ne deviendra un outil d'évaluation que si l'on développe une série d'éléments (proposés plus loin) qui rendent cette technique utilisable pour un objectif particulier.

8 Ainsi, la technique des cartes mentales a été développée ici pour la comparaison entre un prétest et un post-test pour un même groupe (comparaison intragroupe). Cette même technique pourrait être à la base de divers autres outils spécifiques d'évaluation comme, les comparaisons de groupes différents au même moment (comparaison intergroupes). Il serait alors souhaitable de compléter la méthode par l'utilisation d'autres indices tels la "fluidité » (nombre plus grand de mots de la même catégorie dans un groupe que dans l'autre) ou la « rareté » (originalité des mots qui se retrouvent dans un groupe et pas dans l'autre) (Guilford, 1959).

Nous pensons qu'un outil d'évaluation devrait comporter les éléments du tableau 3 : 
Tableau 3 : Éléments indispensables d'un outil d'évaluation en ErE

\begin{tabular}{|c|c|}
\hline Éléments d'un outil d'évaluation & $\begin{array}{l}\text { Lexemple de cet article : carte mentale intra- } \\
\text { groupe }\end{array}$ \\
\hline $\begin{array}{l}\text { Des indications claires et pertinentes quant au } \\
\text { type d'objectif ErE que l'outil peut (aider à) } \\
\text { mesurer }\end{array}$ & $\begin{array}{l}\text { Étude des changements de conception lors des } \\
\text { prises de conscience, de l'intégration des } \\
\text { connaissances et/ou des changements d'attitude }\end{array}$ \\
\hline $\begin{array}{l}\text { Le cadre d'utilisation de cette évaluation : } \\
\text { - Le public utilisateur de l'outil (les évaluateurs) } \\
\text { - Le public cible (les évalués) } \\
\text { - Le matériel nécessaire } \\
\text { - Le temps/espace } \\
\text { - Le type d'évaluation (Piette et al., 2000) }\end{array}$ & $\begin{array}{l}\text { - formateurs de terrain en ErE, } \\
\text { - public adolescent au minimum } \\
\text { - papier-crayon } \\
\text { - modules courts ( } 2 \times 1 \text { heure + analyse), en classe } \\
\text { - } \text { pour orienter la construction d'un module } \\
\text { et/ou pour faire le bilan sur l'efficacité d'un } \\
\text { module de formation }\end{array}$ \\
\hline $\begin{array}{l}\text { Une présentation claire de la méthode de recueil } \\
\text { des informations }\end{array}$ & $\begin{array}{l}\text { Une feuille vierge, un mot au centre, une invita- } \\
\text { tion à le définir ( }+ \text { un exemple sur un autre sujet) }\end{array}$ \\
\hline $\begin{array}{l}\text { Une présentation du fondement scientifique de } \\
\text { l'outil }\end{array}$ & $\begin{array}{l}\text { Le principe des cartes mentales et l'hypothèse de } \\
\text { Norman sur l'enrichissement (1982) }\end{array}$ \\
\hline $\begin{array}{l}\text { Une présentation de la méthode spécifique } \\
\text { d'analyse des informations recueillies en fonc- } \\
\text { tion des objectifs développés }\end{array}$ & $\begin{array}{l}\text { - la proposition des catégories sémantiques } \\
\text { - le développement des divers indices } \\
\text { (statistiques) appropriés aux objectifs et au } \\
\text { contexte }\end{array}$ \\
\hline
\end{tabular}

\section{Perspectives}

La technique des cartes mentales peut encore prendre deux autres directions qui n'ont pas été explorées dans notre recherche-action.

\section{Allier évaluation et formation}

61 Il est possible de transformer la technique des cartes mentales de sorte que cette évaluation constitue un temps de formation comme le préconise Hadji (1997). Les informations obtenues dans les cartes mentales du prétest comme moyen d'orienter la formation ou la comparaison prétest/post-test pour objectiver l'enrichissement seraient complétées par l'utilisation des cartes mentales dans les séquences d'apprentissage. Nous en proposons quelques-unes.

- Les participants pourraient analyser la carte de leurs pairs selon les méthodes d'analyses décrites ci-dessus en se penchant sur l'essentiel : quelles sont les catégories utilisées, quelles sont les configurations systémiques présentes. Cette analyse pourrait d'ailleurs constituer une façon intéressante d'introduire l'étude de la systémique. Cette correction mutuelle serait un moyen d'enrichissement par interactivité et pourrait être à la base d'un débat relatif à la pertinence des catégories, etc.

- On peut aussi, si l'on préfère un travail plus individuel, remplacer le post-test par une reconstruction, une amélioration directe de sa propre carte mentale faite en prétest.

- Il existe en formation professionnelle, des activités basées sur des constructions de cartes mentales en petit groupe afin d'alimenter un débat entre pairs. Iguanane et coll. (1999) proposent une séquence d'activités en ce sens. Deux étapes sont à souligner : (1) Juste après la construction des cartes mentales du prétest, les animateurs effectuent une lecture de ces cartes. Cette lecture redonne du sens à travers les différents apports qui paraissent parfois 
bien étriqués, même à ceux qui ont construit la carte. (2) À la fin de la formation professionnelle, la création d'une carte post-test a permis la prise de conscience par les participants de leur propre évolution. En effet, il n'est pas rare que lors d'une formation de type "pédagogie active » qui ne comprend pas de transmission importante d'une matière, mais plutôt une réflexion sur la pratique, les participants aient l'impression de n'avoir rien appris. La carte mentale permet la visualisation des concepts développés en mémoire à long terme alors que les participants n'en étaient pas conscients.

\section{Distinguer praxis et connaissance}

62 Au début de l'article, nous proposions d'utiliser les cartes mentales pour évaluer les objectifs d'ErE que sont la compréhension des savoirs ou la prise de conscience de l'environnement, mais aussi pour évaluer les aspects conceptuels des attitudes tels que nous les évoquions. Nous pensons qu'il serait possible de modifier la technique des cartes mentales afin de cerner ce dernier objectif de manière plus spécifique :

- Il s'agirait, tout d'abord, de choisir un mot-stimulus qui ne soit pas un objet à définir, mais une action (agir pour l'environnement; comment j'agis actuellement pour l'environnement? Comment devrais-je agir pour l'environnement?)

- Ce mot, cette expression serait ensuite complétée en réseau, et non en étoile, les verbes d'action étant placés dans des cercles auxquels doivent se rapporter les autres objets ou concepts. Le but est, évidemment, de rendre plus lisible l'organisation des connaissances en vue d'une pratique, c'est-à-dire la réflexion des sujets autour de leur pratique (la praxis).

Dans la suite de nos recherches, nous espérons pouvoir tester ce changement de consigne et de stimulus afin d'obtenir une carte mentale de praxis à laquelle nous opposerions la carte mentale de compréhension.

\section{BIBLIOGRAPHIE}

Albertini, J.M. (1985). Les jeunes, l'économie et la consommation. Bruxelles : Labor.

Bronfenbrenner, U. (1979). The Ecology of Human Development. Cambridge, MA : Harvard University Press.

Gagne, R. M. (1965). The Conditions of Learning. New York : Holt Rinehart \& Winston.

Gardner, H. (1993). Histoire de la révolution cognitive. La nouvelle science de l'esprit. Paris : Payot.

Goffin, L. (1993). Comprendre et pratiquer l'éducation relative à l'environnement. Dans Catalogueguide de l'éducation à l'environnement. Bruxelles : Médiathèque de la Communauté Française de Belgique.

Goffin, L. (1998). L'environnement comme écosociosystème. Dans Populations et développement : une approche globale et systémique. Louvain-la-Neuve : Academia-Bruylant/L'Harmattan. p. 199-230.

Goffin, L. (1999). Pour une recherche en éducation relative à l'environnement « centrée sur l'objet partagé ». Éducation relative à l'environnement : Regards, Recherches, Réflexions, 1, 41-63. 
Goldhaber, D.E. (1988). Psychologie du développement. (Traduit par M.C. Desorcy). Québec : Vigot. Guilford, J.P. (1959). Personality. New York : Mac Graw Hill.

Hadji, C. (1997). L'évaluation démystifiée. Paris : Éditions Sociales Françaises.

Iguanane, J., Marchand, C. et d'Ivernois, J.F. (1999). Les cartes sémantiques, outils de formation. Dans P. Lodewick (dir.), Cartes et relations. Les politiques sociales, 1-2.

Johnson-Laird, P.N. (1983). Mental Models. London, Cambridge : University Press.

Johssua, S. et Dupin, J.J. (1993). Introduction à la didactique des sciences et des mathématiques. Paris : Presses Universitaires de France.

Krathwohl, D., Bloom, B. et Masia, B. (1964). Taxonomie des objectifs. Tome II : Domaine affectif. (Traduction française, 1970). Montréal : Entreprises d'Éducation Nouvelle.

Lafon, R. (1979). Vocabulaire de psychopédagogie et de psychiatrie de l'enfant. Paris : Presses Universitaires de France.

Legrand, E. (1997). Comment les enseignants pensent le contexte : Étude exploratoire du réseau conceptuel de deux enseignants différents. Mémoire de Licence. Liège : Université de Liège.

Lucas, A.M. (1981). The Role of Science Education in Education for the Environment. Journal of Environmental Education, 12 (2), 32-37.

Marchand, C. (1997). Intérêt des cartes sémantiques dans l'éducation du patient. Le bulletin d'éducation du Patient, 19 (4), 33-36.

Norman, D.A. (1982). Learning and Memory. San Francisco : Freeman and Co.

Piette, S.A., Legrand, E., Goffin, L., von Frenckell, M., Croizer, C., Philippet, C. et Wattecamps, J.M. (2000). Le partenariat au service de l'évaluation de projets d'éducation relative à

l'environnement. Éducation relative à l'environnement : Regards, Recherches, Réflexions, 2.

Ragou, P. (2000). L'approche systémique et la modélisation-simulation : analyse critique de la valeur didactique en éducation pour l'environnement. Université Paris Sud Onze, Groupe d'Histoire et de Diffusion des Sciences : Thèse de doctorat.

Sauvé, L. (1994). Pour une éducation relative à l'environnement : éléments de design pédagogique. Montréal/Paris : Guerin/ESKA, collection « le défi éducatif » (Renald Legendre).

UNESCO. (1978). Rapport final. Conférence intergouvernementale sur l'éducation relative à l'environnement. Tbilissi (URSS), 14-26 octobre 1977. Paris : UNESCO.

\section{NOTES}

1. En Belgique, les régents peuvent enseigner dans les 3 premières années du secondaire. Ils sont formés dans des Instituts supérieurs pédagogiques non universitaires de type court (BAC+3). C'est dans ces mêmes instituts que sont formés les instituteurs de maternelle et les instituteurs du primaire.

2. Rappelons qu'en philosophie « concept » se définit par : représentation mentale générale et abstraite d'un objet.

3. Sauvé, (1994), Giolitto et Clary, (1994) ainsi que Morin (1977) ; De Rosnay, Sarr (1987) ; Giordan (1987) et Ragou (1990) pour l'utilité de la recherche didactique sur l'approche systémique » dans Ragou (2000). 
4. «Catégorie ", classe dans laquelle l'on range des objets de même nature et « sémantique ", adj. relatif à la sémantique, au sens, à la signification. Ce concept est donc synonyme de « champ sémantique ", ensemble de mots et de notions qui se rapportent à un même domaine conceptuel ou psychologique. Petit Robert : Dictionnaire de la langue française.

5. Comptabiliser tous les mots dans toutes les cartes et calculer leur fréquence d'apparition est assez fastidieux. Or, la démarche d'analyse la plus habituelle est de regrouper les mots de mêmes catégories sémantiques pour donner un sens à ce corpus de mots! Pourquoi ne pas passer tout de suite aux catégories sémantiques?

6. Il est à noter qu'un classement en terme de Nature-Anthropie (éco-sociosystème) aurait montré une présence des deux catégories sémantiques et une intensité nettement supérieure pour les mots anthropiques, ce qui n'était pas le cas dans la carte du prétest.

7. Schéma en mesures répétées.

8. Le mot «typologie » désigne « la science de l'élaboration des types, facilitant l'analyse d'une réalité complexe et la classification ». Le Petit Robert.

9. L'évaluation d'un enrichissement et d'une plus grande homogénéité se traduit par une augmentation des chiffres qui y correspondent, mais, d'un point de vue scientifique, cet enrichissement devrait être évalué afin de vérifier si cette augmentation est significative. Si le nombre de mots moyen d'une catégorie passe de 10 à 12 , est-ce statistiquement suffisant ? Mais cela nous entraîne vers des hypothèses statistiques plus complexes qui dépassent le cadre d'une utilisation pragmatique des cartes mentales.

\section{RÉSUMÉS}

La technique des cartes mentales est de plus en plus souvent utilisée lors de la formation en éducation relative à l'environnement (ErE) afin de caractériser les conceptions des apprenants. Le GREFE exploite cette technique lors de ses recherches-actions. Comme depuis quelques années, le groupe centre particulièrement ses activités sur les aspects théoriques et pratiques de l'évaluation en ErE, ses chercheurs ont constaté que des progrès devaient et pouvaient être réalisés concernant cette technique. Cet article propose une méthode d'analyse des cartes mentales lors de l'évaluation d'un module de formation utilisant une stratégie: Prétest Traitement - Post-test.

The cognitive maps technique is used in environmental education (EE) in order to characterize the students' conceptions. Our research group (GREFE) adopts this technique in its actionresearch projects. For some years, since our research group concentrates its activities upon theoretical and practical aspects of evaluation in the field of EE, we have concluded that progress should and could be made about this technique. This paper proposes an analysis method of cognitive maps during the evaluation of an EE training course that uses a strategy : Pre-test Treatment - Post-test. 


\section{AUTEUR}

\section{EMMANUEL LEGRAND}

Emmanuel Legrand prépare actuellement une thèse de doctorat en didactique des sciences de l'environnement à la Fondation Universitaire Luxembourgeoise. Il est chargé de recherche concernant l'ErE en milieu scolaire, principalement en formation des maîtres. Il a participé à la coordination de cette revue. 